\title{
LABORATORIO REMOTO PARA LA ENSEÑANZA DE LA PROGRAMACIÓN DE UN ROBOT INDUSTRIAL
}

\author{
REMOTE LABORATORY FOR TEACHING INDUSTRIAL ROBOT \\ PROGRAMMING
}

\section{LABORATOIRE À DISTANCE POUR L'ENSEIGNEMENT DE LA PROGRAMMATION D'UN ROBOT INDUSTRIEL}

\author{
Carlos Ariza L. \\ Universidad Militar Nueva Granada \\ calosariza30@gmail.com
}

\author{
Darío Amaya H. \\ Universidad Militar Nueva Granada \\ dario.amaya@unimilitar.edu.co
}

(Artículo tipo INVESTIGACIÓN. Recibido el 04/04/2011. Aprobado el 30/05/2011)

\begin{abstract}
RESUMEN
El presente artículo describe la evolución del desarrollo y funcionamiento de un laboratorio remoto para la enseñanza de la automatización. Por medio de una aplicación Web, un software de acceso remoto que permite una conexión multiusuario independiente y archivos que contienen código con comandos del símbolo del sistema. Con esto se logra administrar el laboratorio evitando conflictos de uso entre los usuarios. Con este desarrollo se busca incrementar el tiempo de productividad y eliminar las restricciones de espacio y tiempo para la utilización de un robot manipulador de tipo industrial.
\end{abstract}

Palabras clave

Laboratorio Remoto, Robótica, programación.

\section{ABSTRACT}

This article presents the evolution of the development and operation stages of a remote laboratory for automation teaching. By means of a web application, remote access software that enables independent multi-user access and files that contain code with commands of system prompt, it is achieved the management of the laboratory avoiding conflicts between users. This development is intended to increase productivity and to eliminate time-space constraints for the use of an industrial-type manipulator robot.

\section{Keywords}

Remote laboratory, Robotics, Programming.

\section{RÉSUMÉ}

Cet article décrit l'évolution du développement et fonctionnement d'un laboratoire à distance pour l'enseignement de l'automatisation. Au moyen d'une application Web, un logiciel d'accès à distance qui permet un lien multi-utilisateur indépendant et fichiers qui ont code avec instructions du symbole du système on réussit gérer le laboratoire en évitant des conflits d'usage entre les utilisateurs. Avec ce développement on recherche d'augmenter le temps de productivité et éliminer les restrictions de espace et temps pour l'utilisation d'un robot manipulateur de type industriel.

\section{Mots-clés}

Laboratoire à distance, Robotique, Programmation. 


\section{INTRODUCCIÓN}

La educación a distancia en general ha tenido gran evolución en los últimos años gracias a los múltiples avances de las tecnologías de la información y las comunicaciones TIC's. Estos avances han permitido el desarrollo de aplicaciones Web que facilitan el acceso de manera remota a los recursos físicos de los laboratorios, dando lugar al concepto de "laboratorio remoto". Este tipo de laboratorio utiliza la visualización de video a través de la Web, permitiendo a los usuarios interactuar con los dispositivos y equipos reales sin estar en contacto directo con ellos, generando apoyo en los procesos de formación.

Actualmente, los laboratorios remotos son un recurso de gran importancia en la educación como es el caso de la ingeniería, y últimamente se ha visto la necesidad de incorporarlos en la educación a distancia debido a que son parte fundamental en la formación de estudiantes, ya que permiten reforzar el conocimiento adquirido de manera teórica a través de la práctica [1], [2]. Por esto se han desarrollado diversos tipos de laboratorios a distancia, como los laboratorios virtuales y ahora los laboratorios de acceso remoto, que facilitan la implementación de prácticas sin la restricción de cumplir horarios [3], [4], [8].

Los laboratorios remotos presentan diversas ventajas frente a los laboratorios presenciales. En primer lugar, dan la oportunidad a los estudiantes de realizar prácticas evitando la necesidad de transportarse hasta un determinado recinto, y por otra parte, pueden tener acceso a los equipos sin restricciones de tiempo, lo que facilita les facilita la realización de prácticas adicionales para reforzar y adquirir una mayor comprensión de los conceptos aprendidos, además de adquirir mayores habilidades en el manejo de los equipos de laboratorio [5], [7].

Existen numerosas aplicaciones para los laboratorios remotos entre las que se encuentra la enseñanza de la robótica y la Mecatrónica. Un ejemplo son los cursos de control en los que los alumnos pueden aprender el diseño de controladores en tiempo real -entre otras aplicaciones-, observando el comportamiento y la respuesta de los equipos. Estos procesos se describen en [6] y [2].

A nivel mundial se han implementado laboratorios remotos que permiten el aprendizaje de diversas prácticas. En la universidad del sur Australia -UniSAse desarrolló el laboratorio a distancia NetLab, que les permite a los estudiantes realizar circuitos electrónicos utilizando diversos elementos reales, como resistencias, capacitores e inductores [9]. En la Universidad Europea de Madrid se desarrolló el laboratorio remoto LABNET, que permite realizar experimentos de control de estabilización de barcos, nivel y temperatura en lazos cerrado o abierto, utilizando controladores predefinidos [10]. Este desarrollo cuenta con un sistema que genera informes sobre los resultados de los experimentos, permitiendo la visualización de las variables involucradas [8].
Con base en los laboratorios remotos existentes, en la Universidad Militar Nueva Granada se desarrolló una plataforma que permite la realización de prácticas de robótica y programación de PLCs de manera remota utilizando un robot manipulador modelo RV-E2, cuya descripción y comportamiento se describe en este artículo.

\section{PROCEDIMIENTO APLICADO}

A continuación se describen los componentes utilizados para el desarrollo del laboratorio.

\subsection{Descripción de la planta}

La estación controlada en forma remota es un robot manipulador RV-E2 de seis grados de libertad de marca Mitsubishi, con capacidad de carga de $2 \mathrm{Kg}$. Este robot pertenece a un laboratorio de manufactura integrada por computador CIM, en el que se fabrican cilindros de simple efecto compuestos por una base, un actuador, un resorte, y una tapa. El laboratorio CIM está compuesto por una estación de ensamble de cilindros, una banda transportadora -Conveyor-, una planta de revisión, una estación de control numérico computarizado CNC y un almacén de productos terminados. El robot tiene como función trasladar los palletes - portadores de bases de cilindros- entre sus receptores ubicados en las estaciones CNC y Conveyor, con el fin de poder transportar las bases ubicadas en los almacenes a las demás estaciones del laboratorio CIM. En la Fig. 1 se muestra el esquema físico de las estaciones Robot Mitsubishi y CNC.

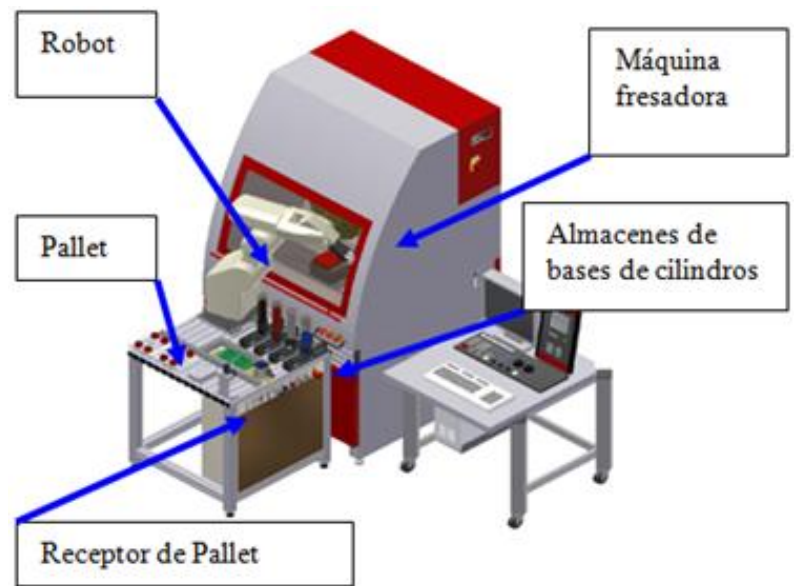

Fig. 1. Robot Mitsubishi y estación CNC (Fuente: Manuales Festo para laboratorio CIM)

El robot puede ser controlado manualmente utilizando un control de comandos - Teach box- que permite moverlo a distintas determinadas, donde se definen sus puntos de movimiento y que son guardados como posiciones predeterminadas. Por medio del software Cosimir Professional se programa la trayectoria del robot de acuerdo con la programación realizada por el usuario. Los siguientes modos se encuentran disponibles para mover al robot con del Teach box:

- Modo joint: Mueve cada eje por separado.

- Modo XYZ: Permite un movimiento en los ejes X, Y y $Z$ del robot. 
- Modo Tool: Permite un movimiento de coordinación de punto en el gripper.

En la Fig. 2 se muestran los distintos sistemas de coordenadas que soporta el Robot de acuerdo con cada eje que lo compone. Este robot cuanta con seis grados de libertad, más la herramienta diseñada específicamente para tomar las piezas que intervienen en el ensamble del producto que se realiza en esta estación de integración de manufactura.
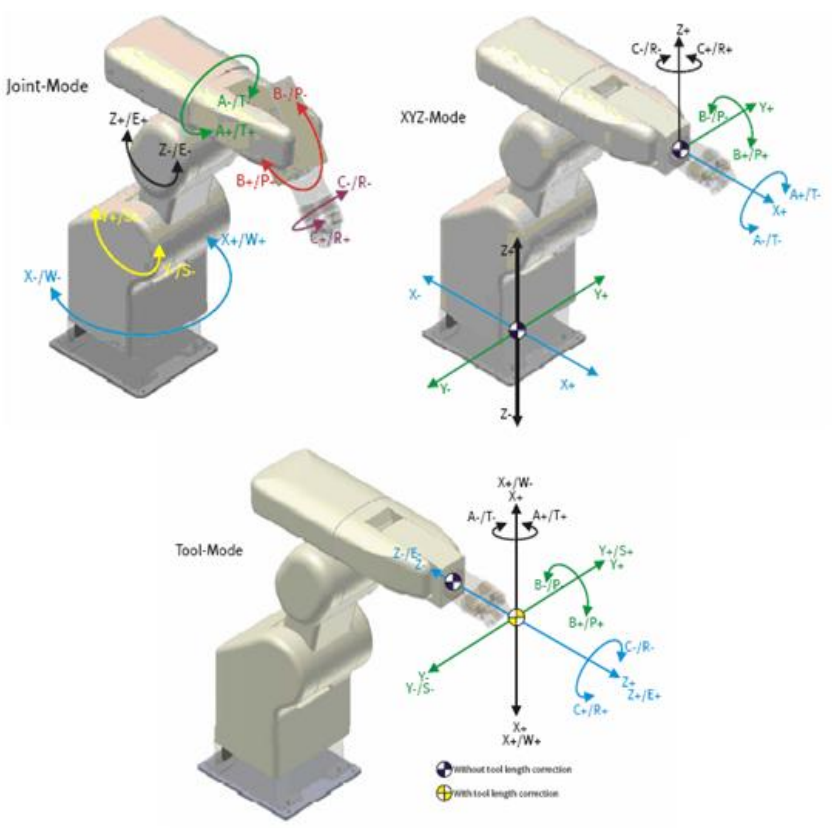

Fig. 2. Sistemas de coordenadas del robot (Fuente: Manuales Festo para laboratorio CIM)

\subsection{Estructura de la aplicación}

Para este desarrollo se diseñó un sistema de comunicación que permite a los usuarios controlar la planta a través de Internet. La arquitectura utilizada se muestra en la Fig. 3. Se realizó una aplicación Web que corre en un servidor instalado en la Universidad, específicamente en el laboratorio CIM, y se instaló una cámara que transmite el video de la estación de trabajo. El usuario accede a la aplicación desde cualquier navegador web, cumpliendo los requisitos de identificación establecidos.

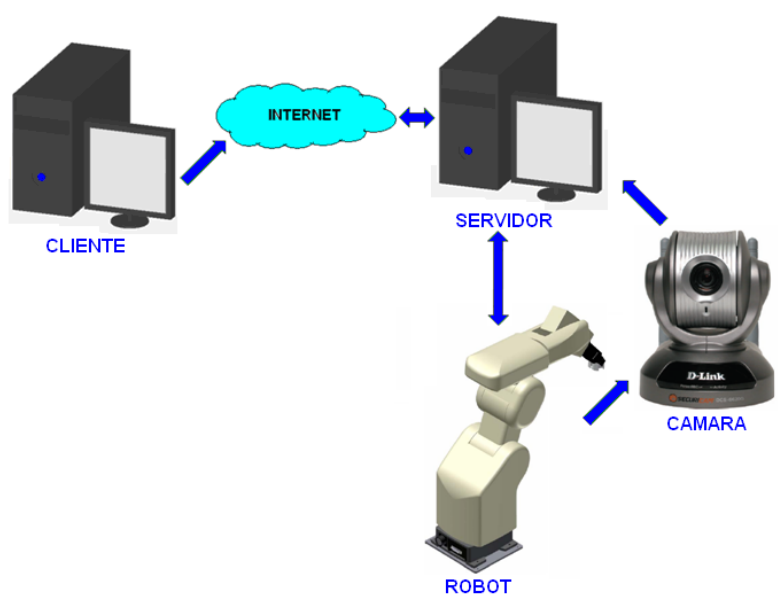

Fig. 3. Conexiones entre el cliente y la planta (Fuente: Manual de la cámara DLINK 66-20 G)
El usuario ingresa a la aplicación Web desde una terminal remota, posteriormente debe identificarse para que el sistema le habilite una sesión que le permita acceder al control de la cámara, la cual puede mover en dos ejes y calibrar la visualización, con lo que puede observar claramente lo que está sucediendo en el proceso. Además, el usuario tiene acceso al programa para desarrollar los algoritmos de control del robot.

Para la implementación del sistema de comunicación se empleó el software REMOTO1 desarrollado mediante la implementación de las siguientes herramientas.

Servidor remoto: Sun Java System Application Server Platform Edition. La aplicación web fue desarrollada utilizando la herramienta NetBeans, y se creó una página principal para facilitar la autenticación de los estudiantes y profesores mediante un nombre de usuario y una contraseña. En otra página los estudiantes pueden realizar la reserva del espacio para trabajar a través del acceso remoto con el laboratorio, en la que el sistema le presenta los espacios libres a escoger en franjas de dos horas. Además, le asigna una contraseña de acceso que le permite el ingreso al momento de realizar su práctica. En otra página puede administrar el sitio Web, y los docentes pueden registrar a los estudiantes que desean tener acceso al Laboratorio, de la misma manera permite identificar a qué Universidad pertenece el estudiante y cuál es el nombre de la asignatura. También se define cuáles los profesores pueden administrar la aplicación.

Servidor de bases de datos: MySQL Server. Toda la información dinámica de la aplicación Web está almacenada en una base datos donde se registran los datos de los estudiantes, profesores, asignaturas, claves de acceso, entre otras.

Software de acceso remoto: Winconnect XP. Permite la conexión de múltiples usuarios al servidor por medio de sesiones independientes del tipo RDP -Protocolo de Escritorio Remoto-, que no interfieren entre sí en cuanto al control del ratón y del teclado. Esto es útil debido a que el servidor se puede aprovechar tanto de manera presencial como remota, además de la posibilidad de utilizar sesiones de usuario independientes en las que se utilicen distintos tipos de software.

\subsection{Visualización de prácticas}

Para este desarrollo se adquirieron cámaras IP DLINK 66-20 G, las cuales permiten una visualización con amplia libertad para los usuarios, debido a que permiten la rotación en dos ejes y acercamientos zoom óptico de 10X. La cámara DLINK 66-20 G se muestra en la Fig. 3. Además, con este tipo de cámaras se pueden realizar grabaciones a través de la detección de movimiento, lo que facilita que los docentes puedan tener un registro en video de la práctica realizada por los usuarios, lo cual se convierte en un instrumento adicional que le permite al docente 
realizar una evaluación más cercana en aspectos como el manejo de equipos, estrategias en la solución de problemas entre otras.

Para la utilización de la cámara se crearon sesiones de administrador y de estudiante. La sesión de administrador permite la configuración total de la cámara, mientras que la sesión de estudiante permite únicamente moverla a distintas posiciones y realizar acercamientos para detallar el funcionamiento de los equipos e instrumentos.

\subsection{Administración del laboratorio}

Para la administración del laboratorio se utilizaron archivos de extensión .bat, que ejecutan instrucciones desde consola, permitiendo así tener control sobre el sistema operativo. Los archivos fueron:

Contraseñas: Permite la creación de contraseñas en forma aleatoria, las cuales serán dadas a los usuarios al momento de realiza la reserva del laboratorio. Además crea un archivo de texto con su contenido.

Temporizador de contraseñas: Permite la ejecución del archivo Contraseñas a determinadas horas del día cada dos horas. Que es el tiempo que tiene el usuario para realizar las actividades correspondientes de su práctica.

Temporizador de sesiones: Tiene como función revisar la hora actual y reiniciar las sesiones a determinadas horas.

Ejecutar al inicio: Comienza la ejecución del temporizador de sesiones, lo que sucede cuando el usuario inicia la sesión para realizar su práctica de laboratorio.

Con el fin de que los usuarios puedan conocer las contraseñas exclusivamente en los horarios registrados en el cronograma de la aplicación Web, los archivos de texto, creados mediante el archivo Contraseñas, se muestran en la página Web de usuarios únicamente cuando el Id del usuario, la fecha y la hora programadas coinciden con las actuales. Además de las contraseñas aparecen links con las direcciones de las cámaras y del escritorio remoto. La página Web de usuario se muestra en la Fig. 4.

Seleccione el laboratorio al que desea ingresar

Laboratorios: \begin{tabular}{lll} 
CIM & $\checkmark$ & Ver Contraseña \\
\hline
\end{tabular}

Contraseña: 28293654

Visualizar Cámara

Visualizar Cámara 2

Acceso Remoto

Fig. 4. Página Web de usuario (Fuente: Autores)

\section{RESULTADOS Y ANALISIS}

Con el fin de comprobar la utilidad del acceso remoto se realizó una práctica de laboratorio. La idea de la práctica era que desde un acceso remoto el estudiante pudiera realizar la programación del robot, de tal manera que definiendo una trayectoria de movimiento y controlando la apertura y cierre de la pinza de agarre, el robot pudiera realizar la manipulación de tres diferentes piezas que deben ser colocadas una detrás de otra.

Como inicio del laboratorio se definieron unas posiciones fijas por las cuales se debe establecer la trayectoria del robot, esto se realizó de manera local en las instalaciones del laboratorio físico. Para esto se utilizó el control de comandos del robot, es decir el teach box. Las diferentes posiciones establecidas para el movimiento del robot, se muestran en la Fig. 5. La estación de trabajo utilizada para la práctica ofrece la posibilidad de configuraciones de piezas, que pueden colocarse como problemas que el estudiante debe resolver; de esta manera el docente cuenta con un potente recurso que le permite evaluar la destreza de los estudiantes en la solución de un problema que requiere la programación de robots de tipo industrial.

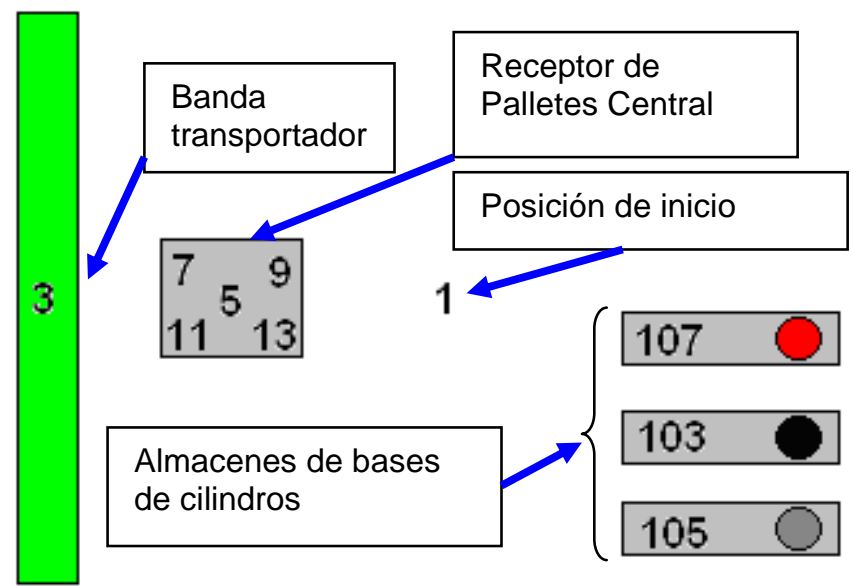

Fig. 5. Posiciones predeterminadas insertadas en el teach box (Fuente: Autores)

Para esta práctica se estableció la posición 1 como referencia del robot, y que es el inicio de la trayectoria de movimiento, la posición 3 lleva el robot hasta la banda transportadora desde donde toma una de las piezas. Las posiciones 103, 105, 107 le permiten al robot tomar los otros dos elementos del ensamble. De acuerdo con los requerimientos, el algoritmo ejecutado por el robot le permitirá seleccionar dos de los tres productos existentes para completar el producto final. Las posiciones $5,7,9,11$ y 13 permiten que el robot realice la manipulación de los palletes.

El estudiante puede realizar el ingreso al sitio accediendo desde cualquier navegador Web a la dirección IP donde se encuentra instalada la aplicación. Una vez el ingreso es satisfactorio ingresa a la página web que se presenta en la Fig. 6, donde debe identificarse para acceder a las otras opciones que provee el laboratorio remoto. 


\section{LABORATORIO REMOTO DE AUTOMATIZACION}

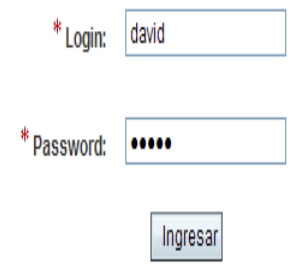

Fig. 6. Página de registro (Fuente: Autores)

Una vez el usuario consiga identificarse con éxito, es llevado a una página que le permite realizar el registro de la reserva del laboratorio remoto. La página le permite ver fechas y horas en las cuales está reservado por otro usuario, y cuáles fechas y horas están disponibles. Con esta información el usuario podrá realizar la programación de sus actividades estipulando la fecha y la hora en la cual va a realizar su práctica. Si la reserva es registrada como exitosa por el sistema, este le muestra un mensaje con los datos de su reserva. La presentación de la página de reserva del laboratorio para el usuario se muestra en la Fig. 7.

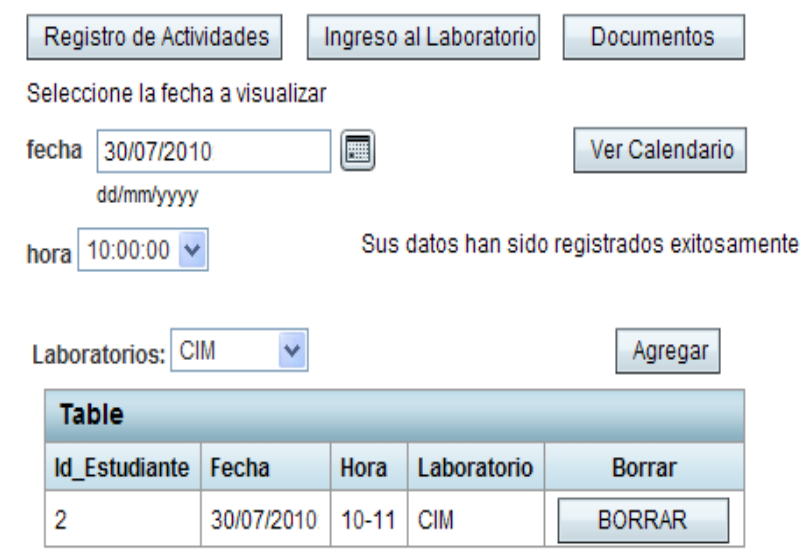

Fig. 7. Página de reserva del laboratorio (Fuente: Autores)

En el día y hora seleccionados para realizar la práctica, nuevamente se ingresa a la página Web de usuario y en la sección Ingreso al laboratorio se selecciona el laboratorio CIM y se presiona el botón Ver Contraseña. Esta contraseña la entrega el sistema y le permite al usuario acceder a la siguiente página que le permite el acceso al laboratorio. Además, se habilita el acceso a través de dos enlaces a las dos cámaras disponibles para observar la dinámica de la práctica, el usuario puede realizar la selección de una de ellas, la otra permanecerá deshabilitada. También, se habilita un enlace de acceso remoto al servidor y a la visualización del laboratorio. La sección de ingreso al laboratorio se observa en la Fig. 8.

Para acceder a una sesión remota en el servidor en la página de acceso remoto se presiona continuar, y posteriormente en la pantalla de registro de sesión de Windows se escribe como ID el nombre del laboratorio CIM, y como contraseña la que aparece en la Fig. 8.

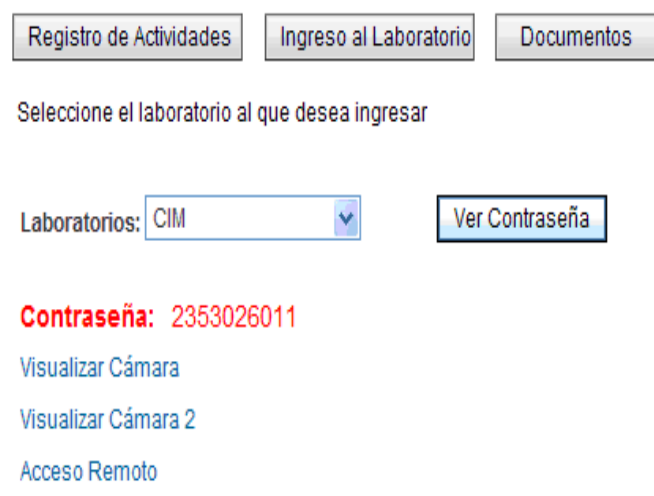

Fig. 8. Ingreso al laboratorio (Fuente: Autores)

Una vez realiza la entrada a la sesión de acceso remoto, el usuario ingresa al servidor donde encuentra las herramientas de desarrollo para realizar las actividades del laboratorio. Una de estas herramientas es el software Cosimir Professional, que le posibilita al usuario desarrollar los algoritmos que puede correr en el controlador del robot para generar las trayectorias a través de los puntos que fueron grabados con anterioridad; todo esto con el propósito de realizar el ensamble del producto, que es el objetivo de la práctica. Accediendo al programa Cosimir se crea un nuevo programa especificando que el tipo de robot es RV-E2.

Posteriormente se importan al programa de Cosimir las posiciones que fueron establecidas para el movimiento del robot, y que actualmente se encuentran en el teach box. Se establece una secuencia de comandos que permite desarrollar el algoritmo que se muestra en la Fig. 9.

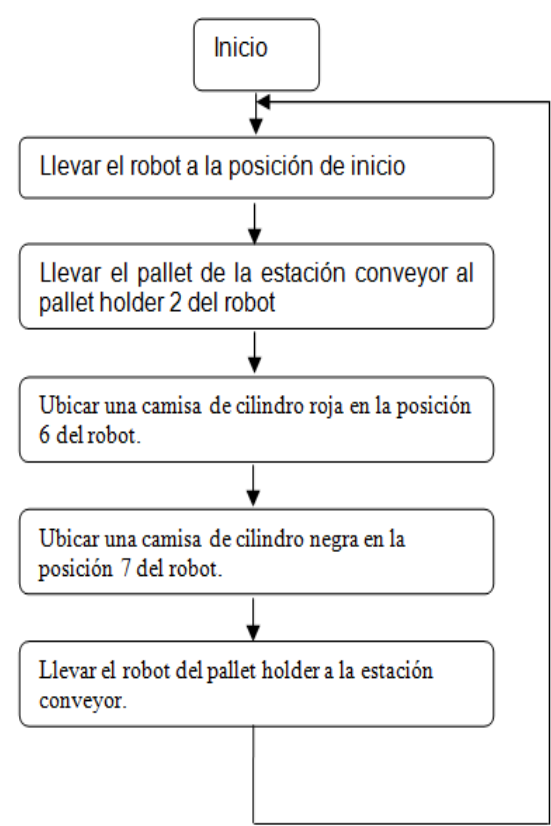

Fig. 9. Algoritmo desarrollado (Fuente: Autores)

Este algoritmo fue programado en la interface de comandos de Cosimir. Además se estableció un ambiente de simulación que le permite al usuario observar la dinámica del proceso al ejecutar su algoritmo, de esta manera se evita una programación 
errónea que pueda causar daños en el robot, ya que un comando equivocado puede hacer que este realice un movimiento por fuera del espacio de trabajo planeado.

El programa, realizado en Cosimir profesional, se observa en la Fig. 10. En la sub-ventana derecha se observan los comandos de programación del robot, en un lenguaje propietario para robots del tipo RV-E2. Con estos comandos se realiza la codificación del algoritmo diseñado para desarrollar la actividad propuesta. Básicamente la codificación lo que hace es dar las órdenes para que el robot recorra, en un determinado orden, los puntos que fueron grabados con anterioridad.

\begin{tabular}{|c|c|c|c|c|}
\hline Aciurclivos de proge..., & : & IC:ARCHVOS DE PROC & GRMALCoSURP PR... . . & (9.... \\
\hline & No & Postion & Dirention & 5 SP $25 ;$. A \\
\hline & 1 & $301.4,-291.8,589.2$ & $131,-7, \quad 45, \mathrm{R}_{f}, \mathrm{~A}_{f} \mathrm{~N}_{f} \mathrm{O}$ & $10 \quad 101$ \\
\hline & 2 & $132,2,-682,3,279.8$ & $135,-5,40, R, 1, F, 0$ & $30 \quad 1153$ \\
\hline & 3 & $132.2,-662.3,198.9$ & 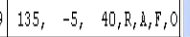 & 40 TI 10 \\
\hline & 4 & $125.6,-470.2,246.3$ & $135,-5,40, R, 1, F$ & $\begin{array}{lll}30 & 0 \\
60 & 4 T & 10\end{array}$ \\
\hline & 5 & $125,6,-470.2,69.2$ & $135,-5, \quad 40, R, \mathbb{L}, \mathbb{F}, 0$ & $70 \mathbb{W L} 2, \mathrm{C}$ \\
\hline & 6 & $92,9,-527,3,234,6$ & $6135,-5, \quad 40, R, L, F, 0$ & $\begin{array}{lll}30 & 10 & 1, C\end{array}$ \\
\hline & 7 & $92.9,-527.3,72.0$ & $135,-5,40, R, R, R, C$ & 100 殁 5,6 \\
\hline & 8 & $92,4,-412,8,231,0$ & $135,-5, \quad 40, R_{t}, \mathbb{A}_{1}, 0$ & 110 TI 10 \\
\hline & & $92,4,-412,8,71,4$ & $135,-5,40, R, 4, F, 0$ & $120 \mathrm{GO}$ \\
\hline & 10 & $159.1,-526.8,207.7$ & $7135, \quad-5, \quad 40, R, 1, F, 0$ & 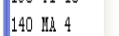 \\
\hline & 11 & $159.1,-526.8,72.0$ & $135,-5,40, R, 1, \mathbb{R}, 0$ & 150101 \\
\hline & 12 & $157,9,-412,8,233.3$ & $135,-5,40, R, 1, F, 0$ & \\
\hline & 13 & $157,9,-412,8,72,0$ & $135,-5,40, R, 1, F, 0$ & 180 TI 10 \\
\hline & 8 & $7 n 4-687+7773$ & $133 \quad-7 \quad 41 \mathrm{~B}$ & 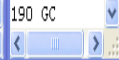 \\
\hline
\end{tabular}

Fig. 10. Programa en Cosimir Professional (Fuente: Autores)

En la ventana central el usuario puede observar la coordenada de cada posición a la que puede moverse al robot y en qué posición está actualmente. Además presenta cuál es la orientación de la pinza de agarre y si está abierta o cerrada. En la parte izquierda de la interface, el usuario puede desarrollar un ambiente de simulación del proceso donde el robot está interactuando. Puede colocar la estructura del robot y también la banda transportadora, el pallet y otros elementos que le permite al usuario realizar una simulación y establecer que la práctica funciona de manera correcta antes de descargar la aplicación al controlador del robot.

Finalmente se descarga el programa al controlador del robot y ejecutarlo a través de la aplicación Cosimir. En la página web de la aplicación se presenta el acceso al control de la cámara, donde se logra observar con detalle el desarrollo de la práctica. Se puede ver cómo ejecuta el robot el algoritmo, cómo recorre los puntos programados para su trayectoria y la manera como ensambla el producto final, el objetivo final de la práctica. La página Web de la aplicación se muestra en la Fig. 11.

Por medio de esta práctica se consiguió comprobar la funcionalidad del laboratorio a través del acceso remoto. Los estudiantes optimizaron el tiempo ya que no tuvieron necesidad de desplazarse hasta las instalaciones de la Universidad; realizaron la práctica en un horario flexible de acuerdo con sus ocupaciones cotidianas. Pudieron realizar varias pruebas y observar a través de la cámara puntos de visualización difíciles de alcanzar cuando se está realizando la práctica de manera presencial.

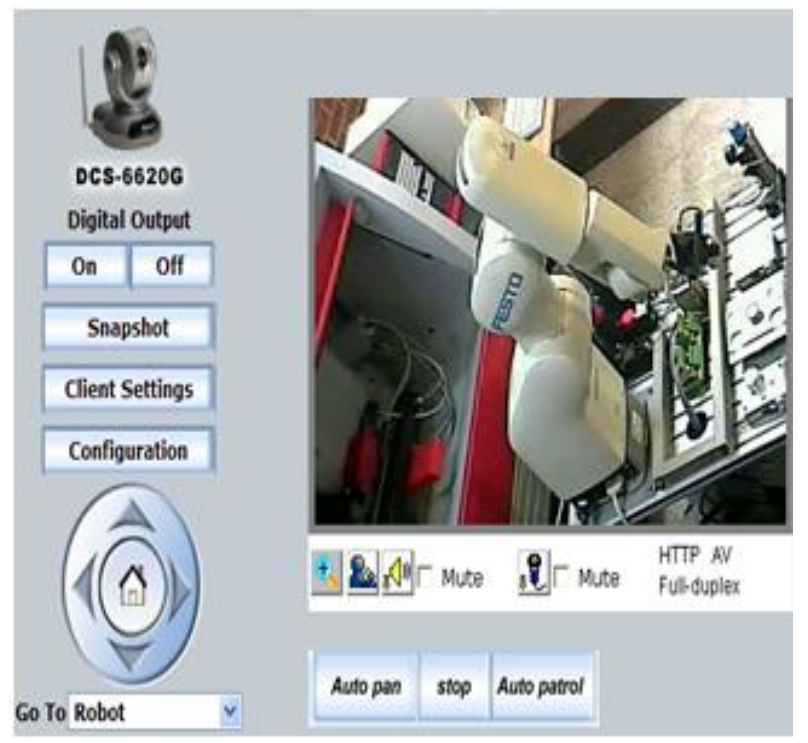

Fig. 11. Página Web para la visualización del laboratorio (Fuente: Autores)

El sistema permitió grabar la práctica realizada, con lo que el docente puede analizar el video para comprobar la dinámica que tuvieron los alumnos al realizar el laboratorio, esto le ayuda a tener más elementos de juicio para realizar su evaluación, ya que le permite evaluar la estrategia utilizada y el tiempo empleado en la realización de la práctica.

Para la Universidad fue un logro importante el que los estudiantes utilizaran los recursos de este laboratorio, ya que le ofreció la posibilidad de que un equipo costoso pueda servir para realizar las actividades de laboratorio de varios estudiantes.

El poder realizar grabaciones de la práctica ayuda a tener seguridad en el manejo de los equipos, pues permite establecer el uso que los estudiantes le dan a los elementos del laboratorio, si el robot se programa para realizar movimientos que lo deterioren, o si se utiliza de manera irresponsable algún recurso del laboratorio. Esto obligará al estudiante a utilizar los equipos con responsabilidad.

\section{CONCLUSIONES}

Gracias a la eliminación de las restricciones en tiempo y espacio que tienen las aplicaciones a distancia se puede optimizar la utilización de los equipos de laboratorio, los cuales tienen un costo elevado y es difícil que una institución académica pueda contar con varios de ellos para la realización de prácticas de apoyo académico. 
Con la utilización de esta herramienta la Universidad podrá optimizar sus recursos de laboratorios, así como humanos, ya que no va tener la necesidad de nombrar un funcionario para estar en frente del laboratorio todo el tiempo.

En el futuro se espera integrar la estación CNC con el robot para realizar prácticas remotas más completas y ofrecerles a los usuarios un mejor servicio.

\section{REFERENCIAS}

[1] M. Auer, A. Pester, D. Ursutiu \& C. Samoila. "Distributed Virtual and Remote Labs in Engineering". Proceedings of the ICIT'03 International Conference on Industrial Technology, Maribor, Slovenia, Dec. 10-12, 2003.

[2] M. Domínguez, J. Fuertes, P. Reguera, A. Diez, A. Robles \& J. Sirgo. "Estrategias docentes colaborativas basadas en la utilización de laboratorios remotos vía Internet". Proceedings of 14 Congreso Universitario de Innovación Educativa en las Enseñanzas Técnicas EUITI de Gijón. Gijón, España, Sep. 27-29, 2006.

[3] C. Ibarra, S. Medina \& A. Bernal. "Implementación de un Laboratorio Virtual para el Estudio de dispositivos Electrónicos". Revista Iberoamericana de Tecnología en Educación y Educación en Tecnología, No. 2, pp. 62-70, Julio, 2007.

[4] E. Lindsay, D. Liu, S. Murray \& D. Lowe. "Remote laboratories in Engineering Education: Trends in Students' Perceptions". Proceedings of the 2007 AaeE Conference, Melbourne, Australia, Dec. 9-13, 2007.
[5] M. Proske \& C. Trodhandl. "Anytime, Everywhere Approaches to Distance Labs in Embedded Systems". Proceedings of International Conference on Information \& Communication Technologies ICTTA 2006, Damascus, Syria, Apr. 24-28, 2006.

[6] L. Rosado \& J. Herrero. "Nuevas Aportaciones Didácticas de los Laboratorios Virtuales y Remotos en la Enseñanza de la Física". Proceedings of The 3rd International Conference on Multimedia and Information and Comunication Technologies in education, Caceres, Spain, June 8-10, 2006.

[7] C. Salzmann \& D. Gillet. "Challenges in Remote Laboratory Sustainability". Proceedings of the International Conference on Engineering Education, ICEE'07, Coimbra, Portugal, Sep. 3-7, 2007.

[8] J. Zhang, A. Ball, M. Clare \& W. Extine. "Design of a Real-Time Remote-Access Engineering Laboratory Using Integrated Web Service and Wireless Technology for Distance Learners". World Transactions on Engineering and Technology Education, Vol. 4, No. 2, pp. 231-234, 2005.

[9] Z. Nedic, J. Machotka \& A. Nafalski. "Remote Laboratory NetLab for Effective Interaction with Real Equipment Over the Internet". Proceedings of IEEE Conference Human Systems Interaction (HSI '08), Kraków, Poland, pp. 846-851, May 25-27, 2008.

[10] N. Aliane, A. Martínez, A. Fraile \& J. Ortiz. "LABNET: Laboratorio remoto para control de procesos". Actas de las XI Jornadas de Enseñanza Universitaria de la Informática, JENUI 2005, Madrid, España, Jul. 13-15, 2005. 